\title{
Observability of Switched Linear Systems in Continuous Time*
}

\author{
Mohamed Babaali and George J. Pappas \\ Electrical and Systems Engineering, \\ University of Pennsylvania, \\ Philadelphia, PA USA \\ \{babaali, pappasg\}@grasp.cis.upenn.edu
}

\begin{abstract}
We study continuous-time switched linear systems with unobserved and exogenous mode signals. We analyze the observability of the initial state and initial mode under arbitrary switching, and characterize both properties in both the autonomous and non-autonomous cases.
\end{abstract}

\section{Introduction}

The general model being considered here i: 1

$$
\begin{aligned}
& \dot{x}_{t}=A\left(r_{t}\right) x_{t}+B\left(r_{t}\right) u_{t} \\
& y_{t}=C\left(r_{t}\right) x_{t}+D\left(r_{t}\right) u_{t}
\end{aligned}
$$

where $x_{t} \in \mathbb{R}^{n}, u_{t} \in \mathbb{R}^{m}$ and $y_{t} \in \mathbb{R}^{p}$, and where $A(\cdot), B(\cdot)$ and $C(\cdot)$ are real matrices of compatible dimensions. The input signals $u:[0, \infty) \rightarrow \mathbb{R}^{m}$ are assumed to be analytic. The exogenous, yet unobserved, mode (or switching) signal

$$
r:[0, \infty) \rightarrow Q \triangleq\{1, \ldots, s\}
$$

is furthermore assumed to be right-continuous and to assume only a finite number of jumps in any finite interval of $[0, \infty)$, so that all trajectories of vectorvalued variables are well defined and infinitely right-differentiable over $[0, \infty)$. Note that Zeno behaviors can thus not occur, even though no minimum separation between consecutive switches (or minimum dwell time) is imposed.

While observability is well understood in classical linear system theory [14, it becomes more complex in the switched case. One reason is that the switching gives rise to a richer set of problems. First, the discrete modes may or may not be observed, giving rise to two sets of problems. Second, in the latter case,

\footnotetext{
* This work was supported by NSF CAREER Grant 0132716.

${ }^{1}$ For notational convenience, we have chosen to subscript time: We will denote the value of some signal $x$ at time $t$ by $x_{t}$ instead of the standard $x(t)$, while $x$ and $x_{\left[t, t^{\prime}\right]}$ will denote the whole signal and its restriction to $\left[t, t^{\prime}\right]$, respectively.
} 
since one may also want to recover the modes, a distinction must be made between recovering the modes and recovering the states. Moreover, one can no longer decouple observation from control, which makes for the need to distinguish between the autonomous and non-autonomous cases, creating the problem of existence of controls allowing observation. Finally, two sets of problems arise from the fact that one may want the observability properties to hold for either all possible mode signals (i.e. universal problems) or for some mode signal (i.e. existential problems), in which case a characterization of the class of signals may be desired. In this paper, we assume that the mode signals are unobserved (i.e. unknown), and study the mode and state observability properties under arbitrary switching.

Observability of hybrid systems has recently been the center of a great deal of attention. However, most of the resulting literature is not related to the problems under consideration here. For instance, while the work in [6, 11, 12, 15, 23] was carried out in a stochastic setting, the papers [3, 5, 9, 18, 13] studied observability of hybrid linear systems, where the modes depend on the state trajectory, and deterministic discrete-time switched linear systems were considered in 1,21. However, in contrast to classical linear systems, there are differences between the discrete and continuous time cases in switched linear systems, which require them to be studied independently. For example, in continuous-time, taking successive time derivatives of the output allows the current mode to fully express itself in infinitesimal time, i.e. provide all the information it can provide about the current state. It is thus possible to decouple the modes in the known modes case, as we will see later in this paper. However, arbitrary switching removes such a luxury in discrete-time (see, e.g., [1).

Returning to continuous-time switched linear systems, we first report the results for observed switching. First, observability under arbitrary switching has long been known to be equivalent to standard observability of every pair $(A(q), C(q))$ (see, e.g., [8]). However, the existence of a mode signal making the initial state observable, which has proven to be a challenging problem, has only recently been characterized, and shown to be decidable, in [10,19. It was shown to be equivalent to $\mathcal{V}=\mathbb{R}^{n}, \mathcal{V}$ being the minimal subspace of $\mathbb{R}^{n}$ invariant with respect to each $A(q)^{T}, q \in Q$, and containing $\sum_{q \in Q} \operatorname{Im} C(q)^{T}$. Furthermore, a constructive procedure for designing the mode signal $r$ was given in [19], along with an upper bound on the minimum number of switches necessary to achieve observability.

It appears that the unobserved switching case has only been analyzed in [2, 7, 22. In 222, the problem of recovering, simultaneously, the initial mode and state was considered along with the switch detection problem, but for autonomous systems. In [2, sufficient conditions were given for generic final state determinability, which we do not consider here. Finally, in [7, notions of observability and detectability were proposed in the framework of linear switching systems, of which our model is a special case. The authors considered the problem of recovering both the initial state and initial mode for some input, again simul- 
taneously, and the problem of detecting the switches, generalizing the results of 22 to the non-autonomous case.

In this paper, we give linear-algebraic characterizations of mode observability and state observability under arbitrary and unobserved switching. The fact that we analyze them separately not only provides criteria for simultaneous state/mode observability (since such a property is characterized by the intersection of both sets of criteria), but provides some additional insight into the specific problems. In particular, by showing that mode and state observability are not necessary for each other, we relax some of the conditions previously given in the literature.

The outline of this paper is as follows. In Section 2, we establish some notation in order to simplify the subsequent exposition. In Section 3 , we study the initial mode and initial state observability problems for autonomous systems. The same treatment is then repeated in the non-autonomous case in Section 4.

\section{Notation}

Letting $w$ denote a trajectory (or execution) of some system comprising all signals of interest, including inputs, outputs and states, we decompose $w$ into three collections of signals or portions of signals over time segments as $w=$ $\left(w_{d}, w_{o}, w_{r}\right)$, and we say a system $\Sigma=\left\{w_{i}\right\}_{i \in I}$ is

$$
\left(w_{d} / w_{o}\right)-\text { observable }
$$

if $w_{d}$, the "desired" set of quantities, can be uniquely recovered when $w_{o}$ is "observed", while $w_{r}$, i.e. the "rest", is neither observed nor desired. In other words, it means that

$$
\forall w, w^{\prime} \in \Sigma,\left(w_{o}=w_{o}^{\prime} \Rightarrow w_{d}=w_{d}^{\prime}\right) .
$$

By default, the domains of all variables are the full spaces of definition, which is often too restrictive since one may find systems that are not $\left(w_{d} / w_{o}\right)$-observable, and yet exhibit trajectories for which $w_{d}$ can be observed from $w_{o}$. Of course the "golden" solution to the observation problem is to actually determine all such trajectories, i.e., find $\Sigma_{0} \triangleq\left\{w \in \Sigma \mid \forall w^{\prime} \in \Sigma, w_{o}=w_{o}^{\prime} \Rightarrow w_{d}=w_{d}^{\prime}\right\}$, the "observable" subset of trajectories. However, we will take a different approach in this paper, and will instead isolate some components of interest (typically inputs, known or unknown) and either restrict them a priori or ask whether the system is observable for some value or for generic values of those components.

We thus define $\left(w_{d} \in W_{d} / w_{o} \in W_{o} / w_{r} \in W_{r}\right)$-observability as

$$
\forall w, w^{\prime} \in \Sigma,\left(w_{d} \in W_{d}, w_{o} \in W_{o}, w_{r} \in W_{r}, w_{o}=w_{o}^{\prime} \Rightarrow w_{d}=w_{d}^{\prime}\right) .
$$

Note that $w^{\prime}$ in (5) ranges over $\Sigma$ : Indeed, for any execution $w$ to determine $w_{d}$, one needs to rule out $w_{d}^{\prime} \neq w_{d} \wedge w_{0}^{\prime}=w_{o}$ for all $w^{\prime} \in \Sigma$. In particular, restricting, say $w_{r}$, to $\{0\}$ will be denoted " $w_{r}$ " instead of " $w_{r} \in\{0\}$." Moreover, since, any 
two restricting sets being fixed (say $W_{o}$ and $W_{d}$ ), one can compute the largest possible third one (i.e., $\left.W_{r}\right)$ such that the system remains $\left(w_{d} \in W_{d} / w_{o} \in\right.$ $\left.W_{o} / w_{r} \in W_{r}\right)$-observable, we will set to compute it, and we will then say the system is

$$
\begin{aligned}
& \left(w_{d} \in W_{d} / w_{o} \in W_{o} / w_{r}^{*}\right)-\text { observable or } \\
& \left(w_{d} \in W_{d} / w_{o} \in W_{o} / \overline{w_{r}}\right) \text { - observable }
\end{aligned}
$$

according as $W_{r}$ is nonempty or generid 2 (when $w_{r}$ lies in a vector space). Informally, (6) reads "is $w_{d}$ observable from $w_{o}$ for some $w_{r}$ ?", while (7) reads "is $w_{d}$ observable from $w_{o}$ for generic $w_{r}$ ?", and are natural questions to ask when $w_{r}$ is some input to the system. Finally, extending the previous conventions to the case where the three components of $w$ themselves have components, we can summarize what has been studied in the following table.

Table 1. Observability Concepts

\begin{tabular}{|c|c|}
\hline Property & Paper \\
\hline$\left(r_{0}, x_{0} \neq 0 / y, \underline{u}\right)$-observability & {$[22]$} \\
\hline$\left(r_{0}, x_{0} / y, u^{*}\right)$-observability & {$[7]$} \\
\hline$\left(r_{0} / y, \underline{u} / \overline{x_{0}}\right)$-observability & \\
$\left(x_{0} / y, \underline{u}\right)$-observability & This paper \\
$\left(r_{0} / y, u^{*}\right)$-observability & \\
$\left(x_{0} / y, u^{*}\right)$-observability & \\
\hline
\end{tabular}

Finally, we establish the following notational conventions to ease the discussion. First, let $y\left(r, x_{0}, u\right)$ be the output signal $y$ of (1) when the initial state is $x_{0}$, the input signal is $u$ and the mode signal is $r$. For any vector-valued signal $z$, let $z^{(N)}$ denote its $N^{\text {th }}$ right-derivative with respect to time, and let

$$
z^{[N]} \triangleq\left(\begin{array}{l}
z \\
z^{\prime} \\
\vdots \\
z^{(N-1)}
\end{array}\right)
$$

Now, let the $N$-step observability matrix of a mode $q \in Q$ be

$$
\mathcal{O}_{N}(q) \triangleq\left(\begin{array}{l}
C(q) \\
\vdots \\
C(q) A(q)^{N-1}
\end{array}\right)
$$

the $N$-step behavior (Toeplitz) matrix of a mode $q$ be

\footnotetext{
${ }^{2}$ We define a generic subset of a finite-dimensional vector space as a set containing an open and dense subset (here, all such subsets will be complements of finite unions of proper subspaces), and a generic subset of the space $S$ of analytic signals from $[0, \infty)$ to $\mathbb{R}^{m}$ as a set containing a set of signals that can be written $\left\{s \in S \mid s_{t}^{[N]} \in G\right\}$ for some integer $N$, some time $t$, and some generic subset $G$ of $\mathbb{R}^{N m}$.
} 


$$
\Gamma_{N}(q) \triangleq\left(\begin{array}{cccc}
D(q) & \cdots & 0 & 0 \\
C(q) B(q) & \cdots & 0 & 0 \\
C(q) A(q) B(q) & \cdots & \vdots & 0 \\
\vdots & \cdots & D(q) & \vdots \\
C(q) A(q)^{N-1} B(q) & \cdots & C(q) B(q) & D(q)
\end{array}\right),
$$

and define the following mapping as

$$
Y_{N}(q, x, U) \triangleq \mathcal{O}_{N}(q) x+\Gamma_{N}(q) U,
$$

where $U \in \mathbb{R}^{m N}$, so that

$$
y_{t}^{[N]}\left(r, x_{0}, u\right)=Y_{N}\left(r_{t}, x_{t}, u_{t}^{[N]}\right) .
$$

In words, $Y_{N}(q, x, U)$ is the stack of the first $N$ derivatives of the output $y_{t}$ when $r_{t}=q, x_{t}=x$, and $u_{t}^{[N]}=U$.

For further reference, we define the following coupled system parameters

$$
\begin{array}{ll}
A\left(q, q^{\prime}\right) \triangleq\left(\begin{array}{cc}
A(q) & 0 \\
0 & A\left(q^{\prime}\right)
\end{array}\right) & B\left(q, q^{\prime}\right) \triangleq\left(\begin{array}{r}
B(q) \\
-B\left(q^{\prime}\right)
\end{array}\right) \\
C\left(q, q^{\prime}\right) \triangleq\left(\begin{array}{ll}
C(q) & C\left(q^{\prime}\right)
\end{array}\right) & D\left(q, q^{\prime}\right) \triangleq D(q)-D\left(q^{\prime}\right),
\end{array}
$$

and we note that the $N$-step Kalman observability matrix of the pair $\left(A\left(q, q^{\prime}\right)\right.$, $\left.C\left(q, q^{\prime}\right)\right)$ is $\left(\mathcal{O}_{N}(q) \mathcal{O}_{N}\left(q^{\prime}\right)\right)$ and that the behavior matrix of the tuple $\left(A\left(q, q^{\prime}\right)\right.$, $\left.B\left(q, q^{\prime}\right), C\left(q, q^{\prime}\right), D\left(q, q^{\prime}\right)\right)$ is simply $\Gamma_{N}(q)-\Gamma_{N}\left(q^{\prime}\right)$.

Furthermore, we let $\rho(M), \Re(M)$ and $M^{\{1\}}$ denote the rank, the column range space, and a (generalized) $\{1\}$-inverse of any real matrix $M$ (see [4]). A matrix $N$ is a $\{1\}$-inverse of $M$ if $M N M=M$. The pseudo-inverse is thus always a $\{1\}$-inverse, and whenever $M$ is of full column rank, any $\{1\}$-inverse $N$ of $M$ is also a left inverse of $M$ in the sense that $M^{\{1\}} M$ equals the identity matrix. Moreover, $x$ is a solution to the equation $Y=M x$ if and only if $x=M^{\{1\}} Y$ for some $\{1\}$-inverse $M^{\{1\}}$ of $M$. Given a subspace $V$ of $\mathbb{R}^{n}$, we let $P_{V}$ denote the matrix of the orthogonal projection on $V$. Finally, let $\mathcal{A}$ denote the set of analytic signals from $[0, \infty)$ to $\mathbb{R}^{m}$.

\section{Autonomous Systems}

In this section we assume that $u=0$, hence the autonomous case. We start with the important observation that the SLS (11) cannot be $\left(r_{0} / y, \underline{u}\right)$-observable. Indeed, if $x_{0}=0$, then $y=0$ identically for all $r$, and so the measurements give no information about $r_{0}$. We therefore need to lower our expectation on the observability of the initial mode, and relax the previous requirements. We thus consider observability of the initial mode for generic initial states, and define discernibility as follows. 
Definition 1. The mode $q$ is discernible from another mode $q^{\prime}$ if for all $T>0$, whenever $r_{[0, T]} \equiv q$ and $r_{[0, T]}^{\prime} \equiv q^{\prime}$, the set

$$
\left\{x_{0} \in \mathbb{R}^{n} \mid \forall x_{0}^{\prime} \in \mathbb{R}^{n}, y_{[0, T]}\left(r, x_{0}, 0\right) \neq y_{[0, T]}\left(r^{\prime}, x_{0}^{\prime}, 0\right)\right\} .
$$

is generic in $\mathbb{R}^{n}$.

In words, $q$ is discernible from $q^{\prime}$ if, for generic initial states $x_{0}$, one can rule out $q^{\prime}$ when observing $y\left(r, x_{0}, 0\right)$ over $[0, T]$. Before giving a characterization of discernibility, let us establish the following straightforward lemma:

Lemma 1. Let $M$ and $M^{\prime}$ be two real $N \times n$ matrices, and define $V \triangleq \Re(M) \cap$ $\Re\left(M^{\prime}\right)$. Then

$$
\operatorname{dim} M^{-1}(V)=n-\rho\left(\left(M M^{\prime}\right)\right)+\rho\left(M^{\prime}\right),
$$

where $M^{-1}$ denotes the set-valued inverse of $M$.

Proof. The Grassmann relation gives $\operatorname{dim}(V)=\rho(M)+\rho\left(M^{\prime}\right)-\rho\left(\left(M M^{\prime}\right)\right)$, the Rank Plus Nullity Theorem $\operatorname{dim}\left(M^{-1}(V)\right)=\operatorname{dim}(V)+\operatorname{dim} \operatorname{ker}(M)$ and $n=\rho(M)+\operatorname{dim} \operatorname{ker}(M)$, and the lemma follows.

We have:

Proposition 1. A mode $q$ is discernible from $q^{\prime}$ if and only if

$$
\rho\left(\left(\mathcal{O}_{2 n}(q) \mathcal{O}_{2 n}\left(q^{\prime}\right)\right)\right)>\rho\left(\mathcal{O}_{2 n}\left(q^{\prime}\right)\right) .
$$

Proof. Fix $T>0$. We need to show that

$$
\left\{x_{0} \in \mathbb{R}^{n} \mid \forall x_{0}^{\prime} \in \mathbb{R}^{n}, y_{[0, T]}\left(r, x_{0}, 0\right)=y_{[0, T]}\left(r^{\prime}, x_{0}^{\prime}, 0\right)\right\}
$$

is a generic set if and only if (16) holds. Recalling that $\left(\mathcal{O}_{2 n}(q) \mathcal{O}_{2 n}\left(q^{\prime}\right)\right)$ is the Kalman observability matrix of the pair $\left(A\left(q, q^{\prime}\right), C\left(q, q^{\prime}\right)\right)$ and that $y_{[0, T]}\left(r, x_{0}, 0\right)$ $-y_{[0, T]}\left(r^{\prime}, x_{0}^{\prime}, 0\right)$ is its output in free evolution with initial state $\left(\begin{array}{c}x_{0} \\ -x_{0}^{\prime}\end{array}\right)$, we have

$$
y_{[0, T]}\left(r, x_{0}, 0\right)=y_{[0, T]}\left(r^{\prime}, x_{0}^{\prime}, 0\right) \Leftrightarrow\left(\mathcal{O}_{2 n}(q) \mathcal{O}_{2 n}\left(q^{\prime}\right)\right)\left(\begin{array}{c}
x_{0} \\
-x_{0}^{\prime}
\end{array}\right)=0
$$

since $\operatorname{ker}\left(\left(\mathcal{O}_{2 n}(q) \mathcal{O}_{2 n}\left(q^{\prime}\right)\right)\right)$ is $A\left(q, q^{\prime}\right)$-invariant. We can therefore shift our attention to showing that the complement of

$$
v\left(q, q^{\prime}\right) \triangleq\left\{x_{0} \in \mathbb{R}^{n} \mid \exists x_{0}^{\prime} \in \mathbb{R}^{n},\left(\mathcal{O}_{2 n}(q) \mathcal{O}_{2 n}\left(q^{\prime}\right)\right)\left(\begin{array}{c}
x_{0} \\
x_{0}^{\prime}
\end{array}\right)=0\right\}
$$

in $\mathbb{R}^{n}$ is generic if and only if (16) holds. Defining $V\left(q, q^{\prime}\right) \triangleq \Re\left(\mathcal{O}_{2 n}(q)\right) \cap$ $\Re\left(\mathcal{O}_{2 n}\left(q^{\prime}\right)\right)$, noting that $v\left(q, q^{\prime}\right)=\mathcal{O}_{2 n}(q)^{-1}\left(V\left(q, q^{\prime}\right)\right)$, and then using Lemma 11. we get

$$
\operatorname{dim} v\left(q, q^{\prime}\right)=n-\rho\left(\left(\mathcal{O}_{2 n}(q) \mathcal{O}_{2 n}\left(q^{\prime}\right)\right)\right)+\rho\left(\mathcal{O}_{2 n}\left(q^{\prime}\right)\right)
$$

Therefore, we see that $\operatorname{dim}\left(v\left(q, q^{\prime}\right)\right)<n$, thus that its complement is generic, if and only if (16) holds, which completes the proof. 
Theorem 1. The SLS (1) is $\left(r_{0} / y, \underline{u} / \overline{x_{0}}\right)$-observable if and only if every pair of different modes is mutually discernible.

Proof. $\left(r_{0} / y, \underline{u} / \overline{x_{0}}\right)$-observability means that the set

$$
P \triangleq\left\{x_{0} \in \mathbb{R}^{n} \mid \forall r, r^{\prime}, \forall x_{0}^{\prime}, r_{0}^{\prime} \neq r_{0} \Rightarrow y\left(r, x_{0}, 0\right) \neq y\left(r^{\prime}, x_{0}^{\prime}, 0\right)\right\} .
$$

is generic in $\mathbb{R}^{n}$. Letting

$$
Q\left(q, q^{\prime}\right) \triangleq\left\{x_{0} \in \mathbb{R}^{n} \mid \exists r, r^{\prime}, r_{0}=q, r_{0}^{\prime}=q^{\prime}, \exists x_{0}^{\prime}, y\left(r, x_{0}, 0\right) \neq y\left(r^{\prime}, x_{0}^{\prime}, 0\right)\right\},
$$

we get

$$
P=\mathbb{R}^{n} \backslash \cup_{q \neq q^{\prime}} Q\left(q, q^{\prime}\right)
$$

Now, by right-continuity of the mode signals, for every pair $r, r^{\prime}$, there exists $0<T \leq \infty$ such that $r_{[0, T]} \equiv q, r_{[0, T]}^{\prime} \equiv q^{\prime}$, and so $v\left(q, q^{\prime}\right) \subset Q\left(q, q^{\prime}\right)$ (see Proposition 1). On the other hand, $Q\left(q, q^{\prime}\right) \subset v\left(q, q^{\prime}\right)$ follows by considering $r \equiv q$ and $r^{\prime} \equiv q^{\prime}$. Consequently,

$$
P=\mathbb{R}^{n} \backslash \cup_{q \neq q^{\prime}} v\left(q, q^{\prime}\right),
$$

and is generic if and only if each $v\left(q, q^{\prime}\right)$ is a proper subspace of $\mathbb{R}^{n}$, and thus if and only if every pair of modes is mutually discernible.

Example 1. Consider (11), where $s=2, B(1)=B(2)=0, D(1)=D(2)=0$, and where

$$
\begin{aligned}
& A(1)=\left(\begin{array}{ll}
1 & 1 \\
0 & 1
\end{array}\right) \\
& C(1)=\left(\begin{array}{ll}
1 & 0
\end{array}\right) \quad C(2)=\left(\begin{array}{ll}
1 & 2 \\
0 & 3
\end{array}\right) \\
& C\left(\begin{array}{ll}
1 & 0
\end{array}\right) .
\end{aligned}
$$

Then

$$
\left(\mathcal{O}_{4}(1) \mathcal{O}_{4}(2)\right)=\left(\begin{array}{llll}
1 & 0 & 1 & 0 \\
1 & 1 & 1 & 2 \\
1 & 2 & 1 & 8 \\
1 & 3 & 1 & 26
\end{array}\right)
$$

and has rank 3 , while $\rho\left(\mathcal{O}_{4}(1)\right)=\rho\left(\mathcal{O}_{4}(2)\right)=2$. Therefore, it is possible to recover the initial mode for generic initial states. For instance,

$$
y_{0}^{[4]}\left(r_{0}, x, o\right)=\left(\begin{array}{l}
1 \\
2 \\
3 \\
4
\end{array}\right)
$$

could only have been produced by $r_{0}=1$ (with $x_{0}=(1,1)$ ). It is actually possible to recover $r_{0}$ uniquely whenever the second entry of $x_{0}$ is not zero, which constitutes a generic subset of $\mathbb{R}^{2}$. 
We now turn to the study of the ability to recover the initial state $x_{0}$ of the system, based only on the output signal $y$. A first route for that is, first, to recover the initial mode $r_{0}$, and, then, to invert the Gramian to get $x_{0}$. Noting that this can only be done for generic $x_{0}$, we state the following corollary to Theorem 1 .

Corollary 1. The SLS (1) is $\left(\overline{x_{0}} / y, \underline{u}\right)$-observable if every mode is observable and every pair of modes is mutually discernible.

Even though this route may seem to be the natural way to proceed, we will now show that it is neither necessary nor sufficient for $\left(x_{0} / y, \underline{u}\right)$-observability, which is in fact possible. In other words, it is possible to determine the initial state from the output globally, for all mode signals, and without necessarily recovering the modes. To this end, we define joint observability as follows:

Definition 2. Two different modes $q$ and $q^{\prime}$ are jointly observable if for all $T>0$, whenever $r_{[0, T]} \equiv q$ and $r_{[0, T]}^{\prime} \equiv q^{\prime}$,

$$
\forall x_{0}, \forall x_{0}^{\prime}, x_{0} \neq x_{0}^{\prime} \Rightarrow y_{[0, T]}\left(r, x_{0}, 0\right) \neq y_{[0, T]}\left(r^{\prime}, x_{0}^{\prime}, 0\right) .
$$

Note that, in contrast to discernibility, joint observability is symmetric. That two modes are jointly observable means that one can recover the initial state from the output without knowledge of the initial mode. We have:

Proposition 2. $q$ and $q^{\prime}$ are jointly observable if and only if they are both observable (i.e., $\rho\left(\mathcal{O}_{n}(q)\right)=\rho\left(\mathcal{O}_{n}\left(q^{\prime}\right)\right)=n$ ) and the left inverses of their $2 n$-step observability matrices agree on $V\left(q, q^{\prime}\right)$, i.e.

$$
\left(\mathcal{O}_{2 n}(q)^{\{1\}}-\mathcal{O}_{2 n}\left(q^{\prime}\right)^{\{1\}}\right) P_{V\left(q, q^{\prime}\right)}=0
$$

Proof. Assume that $q$ and $q^{\prime}$ are both observable and satisfy (29), and suppose that $y_{[0, T]}\left(r, x_{0}, 0\right)=y_{[0, T]}\left(r^{\prime}, x_{0}^{\prime}, 0\right)$ (with $T>0$ and $r_{[0, T]} \equiv q$ and $\left.r_{[0, T]}^{\prime} \equiv q^{\prime}\right)$. Then, recalling (18), we get

$$
\mathcal{O}_{2 n}(q) x_{0}=\mathcal{O}_{2 n}\left(q^{\prime}\right) x_{0}^{\prime}
$$

Furthermore, $q$ and $q^{\prime}$ being observable, (29) implies that $v\left(q, q^{\prime}\right)=v\left(q^{\prime}, q\right)$ and that $\left(\mathcal{O}_{2 n}(q)-\mathcal{O}_{2 n}\left(q^{\prime}\right)\right) P_{v\left(q, q^{\prime}\right)}=0$, which, in turn, implies that

$$
\mathcal{O}_{2 n}(q) x_{0}=\mathcal{O}_{2 n}\left(q^{\prime}\right) x_{0},
$$

since $x_{0} \in v\left(q, q^{\prime}\right)$. Combining (30) and (31), we get

$$
\mathcal{O}_{2 n}\left(q^{\prime}\right)\left(x_{0}-x_{0}^{\prime}\right)=0,
$$

hence that $x_{0}=x_{0}^{\prime}$ since $q^{\prime}$ is observable.

Conversely, assume that, say $q$, is not observable. Then taking $x_{0} \in \operatorname{ker}\left(\mathcal{O}_{n}(q)\right)$ $\backslash\{0\}$, we get $y_{[0, T]}\left(r, x_{0}, 0\right)=y_{[0, T]}\left(r^{\prime}, 0,0\right)=0$ while $x_{0} \neq 0$, hence that $q$ and $q^{\prime}$ are not jointly observable. Finally, assuming $q$ and $q^{\prime}$ are both observable but that (29) does not hold, we have the existence of $Y \in V\left(q, q^{\prime}\right)$ such that $\left(\mathcal{O}_{2 n}(q)^{\{1\}}-\mathcal{O}_{2 n}\left(q^{\prime}\right)^{\{1\}}\right) Y \neq 0$. Letting $x_{0}=\mathcal{O}_{2 n}(q)^{\{1\}} Y$ and $x_{0}^{\prime}=\mathcal{O}_{2 n}\left(q^{\prime}\right)^{\{1\}} Y$, we have $x_{0} \neq x_{0}^{\prime}$ but $\mathcal{O}_{2 n}(q) x_{0}=\mathcal{O}_{2 n}\left(q^{\prime}\right) x_{0}^{\prime}=Y$, and thus $y_{[0, T]}\left(r, x_{0}, 0\right)=$ $y_{[0, T]}\left(r^{\prime}, x_{0}^{\prime}, 0\right)$ and $q$ and $q^{\prime}$ are not jointly observable. 
A characterization of $\left(x_{0} / y, \underline{u}\right)$-observability follows.

Theorem 2. The SLS (11) is $\left(x_{0} / y, \underline{u}\right)$-observable if and only if every mode is observable and any two different modes are jointly observable.

Proof. $\left(x_{0} / y, \underline{u}\right)$-observability means that

$$
\forall r, \forall r^{\prime}, \forall x_{0}, \forall x_{0}^{\prime}, x_{0}^{\prime} \neq x_{0} \Rightarrow y\left(r, x_{0}, 0\right) \neq y\left(r^{\prime}, x_{0}^{\prime}, 0\right) .
$$

Assume that every mode is observable, that any pair is jointly observable, and that $y\left(r, x_{0}, 0\right)=y\left(r^{\prime}, x_{0}^{\prime}, 0\right)$. First, by right-continuity of both mode signals, there exist $0<T \leq \infty$ and two modes $q, q^{\prime}$ such that $r_{[0, T]} \equiv q, r_{[0, T]}^{\prime} \equiv q^{\prime}$. Then $x_{0}=x_{0}^{\prime}$ is implied by observability of each mode or joint observability of each pair of modes according as $q=q^{\prime}$ or $q \neq q^{\prime}$, by definition.

Conversely, assume that, say $q$, is not observable. Then letting $r=r^{\prime} \equiv q$, and choosing $x_{0} \in \operatorname{ker}\left(\mathcal{O}_{n}(q)\right) \backslash\{0\}$, we have $y\left(r, x_{0}, 0\right) \neq y\left(r^{\prime}, 0,0\right)$ even though $x_{0} \neq 0$. On the other hand, assuming the existence of a jointly unobservable pair $q, q^{\prime}$, letting $r \equiv q$ and $r^{\prime} \equiv q^{\prime}$, there must exist $x_{0} \neq x_{0}^{\prime}$ such that $y\left(r, x_{0}, 0\right) \neq$ $y\left(r^{\prime}, x_{0}^{\prime}, 0\right)$, by definition of joint observability.

Remark 1. In [22], it was established that $\left(r_{0}, x_{0} \neq 0 / y, \underline{u}\right)$-observability was equivalent to the rank- $2 n$ condition

$$
\forall q, q^{\prime} \in Q, q \neq q^{\prime} \Rightarrow \rho\left(\left(\mathcal{O}_{2 n}(q) \mathcal{O}_{2 n}\left(q^{\prime}\right)\right)\right)=2 n .
$$

Since $\rho\left(\left[\mathcal{O}_{2 n}(j)\right]\right) \leq n$ for both $j=q$ and $j=q^{\prime}$, (34) is sufficient for mutual discernibility of $q$ and $q^{\prime}$, and therefore for $\left(r_{0} / y, \underline{u} / \overline{x_{0}}\right)$-observability. In fact, by (20), it is equivalent to

$$
\forall q, q^{\prime}, q \neq q^{\prime} \Rightarrow v\left(q, q^{\prime}\right)=\{0\},
$$

which is the least-dimensional possible subspace of conflict, and hence to $\left(r_{0} /\right.$ $y, \underline{u} / x_{0} \neq 0$ )-observability. What we have thus shown is that it is possible to recover $r_{0}$ even if $v\left(q, q^{\prime}\right) \neq\{0\}$, and we have relaxed (34) into (16) to account for such cases.

As for state observability, it turns out that (34) is not necessary for $\left(x_{0} / y, \underline{u}\right)$ observability, simply because it is not necessary to recover the initial mode in order to infer the initial state when the initial state is not trivial. For instance, the system in Example 1 is $\left(x_{0} / y, \underline{u}\right)$-observable, but does not satisfy (34). Recall that

$$
v(1,2)=\left\{\left(\begin{array}{l}
\alpha \\
0
\end{array}\right) \mid \alpha \in \mathbb{R}\right\} .
$$

If $x_{0} \notin v(1,2)$, then one can uniquely infer $r_{0}$ and recover $x_{0}$, since every mode is observable. However, if $x_{0} \in v(1,2)$, then

$$
y_{0}^{[2]}\left(r, x_{0}, 0\right)=\left(\begin{array}{c}
\alpha \\
\alpha
\end{array}\right) \Rightarrow x_{0}=\left(\begin{array}{l}
\alpha \\
0
\end{array}\right)
$$

for all $r$, hence the claim. 


\section{Non-autonomous Systems}

We now turn to the non-autonomous case, and study both existence and generic problems in $u$. We will show that existence and generic properties will be equivalent for the initial mode observability properties, and that the genericity requirement on $x_{0}$ can actually be waived. We will need the following definition and lemma.

Definition 3. Two different modes $q$ and $q^{\prime}$ are controlled-discernible if for all $T>0$, whenever $r_{[0, T]} \equiv q$ and $r_{[0, T]}^{\prime} \equiv q^{\prime}$, there exists an input $u$ such that

$$
\forall x_{0}, \forall x_{0}^{\prime}, y_{[0, T]}\left(r, x_{0}, u\right) \neq y_{[0, T]}\left(r^{\prime}, x_{0}^{\prime}, u\right) .
$$

In other words, $q$ and $q^{\prime}$ are controlled-discernible if there exists a control making it possible to distinguish them by their outputs.

Lemma 2. The two modes $q$ and $q^{\prime}$ are controlled-discernible if and only if there exists a positive integer $N$ such that

$$
\left(I-P_{N}\left(q, q^{\prime}\right)\right)\left(\Gamma_{N}(q)-\Gamma_{N}\left(q^{\prime}\right)\right) \neq 0,
$$

where $P_{N}\left(q, q^{\prime}\right)$ is the matrix of the orthogonal projection on $\Re\left(\mathcal{O}_{N}(q)\right) \cap \Re$ $\left(\mathcal{O}_{N}\left(q^{\prime}\right)\right)$. Moreover, (38) then holds if and only if $\left(I-P_{N}\left(q, q^{\prime}\right)\right)\left(\Gamma_{N}(q)-\right.$ $\left.\Gamma_{N}\left(q^{\prime}\right)\right) u_{0}^{[N]} \neq 0$.

Proof. First, note that since the inputs $u$ are analytic, we have

$$
\begin{aligned}
& y_{[0, T]}\left(r, x_{0}, u\right)=y_{[0, T]}\left(r^{\prime}, x_{0}^{\prime}, u\right) \\
\Longleftrightarrow & \forall N, y_{0}^{[N]}\left(r, x_{0}, u\right)=y_{0}^{[N]}\left(r^{\prime}, x_{0}^{\prime}, u\right) \\
\Longleftrightarrow & \forall N, Y_{N}\left(q, x_{0}, u_{0}^{[N]}\right)=Y_{N}\left(q^{\prime}, x_{0}^{\prime}, u_{0}^{[N]}\right) .
\end{aligned}
$$

Therefore, $q$ and $q^{\prime}$ are controlled-discernible if and only if there exists $u$ such that

$$
\begin{aligned}
& \forall x_{0}, \forall x_{0}^{\prime}, \exists N, \mathcal{O}_{N}(q) x_{0}+\Gamma_{N}(q) u_{0}^{[N]} \neq \mathcal{O}_{N}\left(q^{\prime}\right) x_{0}^{\prime}+\Gamma_{N}\left(q^{\prime}\right) u_{0}^{[N]} \\
\Longleftrightarrow & \exists N, \forall x_{0}, \forall x_{0}^{\prime}, \mathcal{O}_{N}(q) x_{0}+\Gamma_{N}(q) u_{0}^{[N]} \neq \mathcal{O}_{N}\left(q^{\prime}\right) x_{0}^{\prime}+\Gamma_{N}\left(q^{\prime}\right) u_{0}^{[N]} \\
\Longleftrightarrow & \left(\Re\left(\mathcal{O}_{N}(q)\right)+\Gamma_{N}(q) u_{0}^{[N]}\right) \cap\left(\Re\left(\mathcal{O}_{N}\left(q^{\prime}\right)\right)+\Gamma_{N}\left(q^{\prime}\right) u_{0}^{[N]}\right)=\emptyset \\
\Longleftrightarrow & \left(I-P_{N}\left(q, q^{\prime}\right)\right)\left(\Gamma_{N}(q)-\Gamma_{N}\left(q^{\prime}\right)\right) u_{0}^{[N]} \neq 0 .
\end{aligned}
$$

Equivalence of (44) and (43) follows from the fact that the sets

$$
S_{N} \triangleq\left\{\left(\begin{array}{c}
x_{0} \\
-x_{0}^{\prime}
\end{array}\right) \in \mathbb{R}^{2 n} \mid\left(\mathcal{O}_{N}(q) \mathcal{O}_{N}\left(q^{\prime}\right)\right)\left(\begin{array}{c}
x_{0} \\
-x_{0}^{\prime}
\end{array}\right)+\left(\Gamma_{N}(q)-\Gamma_{N}\left(q^{\prime}\right)\right) u_{0}^{[N]}=0\right\}
$$

are affine subspaces of $\mathbb{R}^{2 n}$ satisfying $S_{N} \subset S_{N^{\prime}}$ if $N>N^{\prime}$, and so $\cap_{N=1}^{\infty} S_{N}=\emptyset$ if and only if $S_{N}$ eventually stabilizes at $\emptyset$. 
Therefore, there exists an input $u$ such that (38) holds if and only if there exists $N$ such that (39) holds, and the set of such inputs then contains the set

$$
\left\{u \in \mathcal{A} \mid u_{0}^{[N]} \in \mathbb{R}^{m N} \backslash \operatorname{ker}\left(\left(I-P_{N}\left(q, q^{\prime}\right)\right)\left(\Gamma_{N}(q)-\Gamma_{N}\left(q^{\prime}\right)\right) u_{0}^{[N]}\right)\right\},
$$

which is generic.

The next result establishes the decidability of the condition given in the previous lemma.

Proposition 3. The two modes $q$ and $q^{\prime}$ are controlled-discernible if and only if

$$
\Gamma_{2 n}(q)-\Gamma_{2 n}\left(q^{\prime}\right) \neq 0
$$

and, equivalently, if (39) is satisfied with $N=4 n$.

Proof. First, let us show that

$$
\begin{aligned}
& \exists N,\left(I-P_{N}\left(q, q^{\prime}\right)\right)\left(\Gamma_{N}(q)-\Gamma_{N}\left(q^{\prime}\right)\right) \neq 0 \\
\Longleftrightarrow & \exists N^{\prime}, \Gamma_{N^{\prime}}(q)-\Gamma_{N^{\prime}}\left(q^{\prime}\right) \neq 0,
\end{aligned}
$$

To see this, note that

$$
\begin{aligned}
& \left(I-P_{N}\left(q, q^{\prime}\right)\right)\left(\Gamma_{N}(q)-\Gamma_{N}\left(q^{\prime}\right)\right) \neq 0 \\
\Longleftrightarrow & \rho\left(\left(\Gamma_{N}(q)-\Gamma_{N}\left(q^{\prime}\right) \mathcal{O}_{N}(q) \mathcal{O}_{N}\left(q^{\prime}\right)\right)\right)>\rho\left(\left(\mathcal{O}_{N}(q) \mathcal{O}_{N}\left(q^{\prime}\right)\right)\right),
\end{aligned}
$$

which clearly proves the implication in (51). On the other hand, necessity in (51) stems from the fact that if $\Gamma_{N}(q)-\Gamma_{N}\left(q^{\prime}\right) \neq 0$, then the rank of $\Gamma_{N}(q)-\Gamma_{N}\left(q^{\prime}\right)$, thus that of $\left(\Gamma_{N}(q)-\Gamma_{N}\left(q^{\prime}\right) \mathcal{O}_{N}(q) \mathcal{O}_{N}\left(q^{\prime}\right)\right)$, grows unbounded in $N$. Therefore, since the rank of $\left(\mathcal{O}_{N}(q) \mathcal{O}_{N}\left(q^{\prime}\right)\right)$ is bounded by $2 n$,

$$
\rho\left(\left(\Gamma_{N}(q)-\Gamma_{N}\left(q^{\prime}\right) \mathcal{O}_{N}(q) \mathcal{O}_{N}\left(q^{\prime}\right)\right)\right)-\rho\left(\left(\mathcal{O}_{N}(q) \mathcal{O}_{N}\left(q^{\prime}\right)\right)\right)
$$

is unbounded.

Now, a straightforward consequence of the Cayley-Hamilton Theorem is that $\Gamma_{N}(q) \neq 0$ for some $N$ if and only if $\Gamma_{n}(q) \neq 0$. Therefore, recalling that $\Gamma_{N}(q)-$ $\Gamma_{N}\left(q^{\prime}\right)$ is exactly the $N$-step behavior matrix of the tuple $\left(A\left(q, q^{\prime}\right), B\left(q, q^{\prime}\right)\right.$, $\left.C\left(q, q^{\prime}\right), D\left(q, q^{\prime}\right)\right)$, we get that (51) holds if and only if $\Gamma_{2 n}(q)-\Gamma_{2 n}\left(q^{\prime}\right) \neq 0$. Moreover, in that case, it is easy to see that $\rho\left(\Gamma_{4 n}(q)-\Gamma_{4 n}\left(q^{\prime}\right)\right)>2 n$, and therefore that the integer expressed in (54) is positive, and thus that $(I-$ $\left.P_{4 n}\left(q, q^{\prime}\right)\right)\left(\Gamma_{4 n}(q)-\Gamma_{4 n}\left(q^{\prime}\right)\right) \neq 0$.

Remark 2. An interesting question is whether the smallest $N^{\prime}$ in (51) could be strictly smaller than the smallest $N$. Equivalently, can the degree of a polynomial input $u^{\prime}$ of smallest degree satisfying 


$$
y(r, 0, u) \neq y\left(r^{\prime}, 0, u\right)
$$

where $r \equiv q$ and $r^{\prime} \equiv q^{\prime}$, be strictly smaller then the degree of a polynomial input $u$ of smallest degree satisfying (38)? The answer is yes, and as an example, take $q=1$ and $q^{\prime}=2$, with $A(1)=B(1)=(1), A(2)=B(2)=(2), C(1)=$ $C(2)=(1)$, and $D(1)=D(2)=0$, and let $u \equiv-1$. Then

$$
y_{0}^{[4]}(r, 0, u)=\left(\begin{array}{c}
0 \\
-1 \\
-1 \\
-1
\end{array}\right) \neq y_{0}^{[4]}\left(r^{\prime}, 0, u\right)=\left(\begin{array}{c}
0 \\
-2 \\
-4 \\
-8
\end{array}\right),
$$

hence (55). However, if $u \equiv \alpha$, then whenever $x_{0}=x_{0}^{\prime}=-\alpha$, we get

$$
y\left(r, x_{0}, u\right)=y\left(r^{\prime}, x_{0}^{\prime}, u\right) .
$$

In fact, it can be verified that the minimum degree of a polynomial $u$ for (38) to hold is 1 , as opposed, obviously, to 0 for (55).

We can now establish the following characterization of $\left(r_{0} / y, u^{*}\right)$-observability and $\left(r_{0} / y, \bar{u}\right)$-observability.

Theorem 3. The following are equivalent.

1. The SLS (1) is $\left(r_{0} / y, u^{*}\right)$-observable.

2. The $S L S$ (1) is $\left(r_{0} / y, \bar{u}\right)$-observable.

3. Every pair of different modes is controlled-discernible.

Proof. $2 \Rightarrow 1$ is obvious.

$1 \Rightarrow 3$ : Assume some pair of modes $\left(q, q^{\prime}\right)$ is not controlled-discernible. Then, by definition, there exists no input $u$ such that $y\left(r, x_{0}, u\right) \neq y\left(r^{\prime}, x_{0}^{\prime}, u\right)$ for all $x_{0}, x_{0}^{\prime}$ when $r \equiv q$ and $r^{\prime} \equiv q^{\prime}$.

$3 \Rightarrow 2$ : We need to show that the set of controls

$$
\mathcal{U} \triangleq\left\{u \in \mathcal{A} \mid \forall r, \forall r^{\prime}, \forall x_{0}, \forall x_{0}^{\prime}, r_{0}^{\prime} \neq r_{0} \Rightarrow y\left(r, x_{0}, u\right) \neq y\left(r^{\prime}, x_{0}^{\prime}, u\right)\right\}
$$

is generic if condition 3. holds. Let us show that it in fact contains

$$
\mathcal{U}_{4 n} \triangleq\left\{u \in \mathcal{A} \mid u_{0}^{[4 n]} \in \mathbb{R}^{4 m n} \backslash \bigcup_{q \neq q^{\prime}} \operatorname{ker}\left(\left(I-P_{4 n}\left(q, q^{\prime}\right)\right)\left(\Gamma_{4 n}(q)-\Gamma_{4 n}\left(q^{\prime}\right)\right)\right)\right\},
$$

which is indeed generic if ever pair of modes is controlled-discernible, by Proposition 3. That $\mathcal{U}_{4 n} \subset \mathcal{U}$ follows from Lemma 2 and from the fact that, since the mode signals are right-continuous, there exists for any $r$ and $r^{\prime}$ a time $T>0$ such that $r_{[0, T]} \equiv q$ and $r_{[0, T]}^{\prime} \equiv q^{\prime}$. 
As for state observability, we have:

Theorem 4. The following are equivalent.

1. The $S L S$ (1) is $\left(x_{0} / y, u^{*}\right)$-observable.

2. The $S L S$ (1) is $\left(x_{0} / y, \bar{u}\right)$-observable.

3. Every mode is observable and every pair of modes is either controlleddiscernible or jointly observable.

Proof. $2 \Rightarrow 1$ is obvious.

$1 \Rightarrow 3$ : If some mode $q$ is not observable, then whenever $x_{0} \in \operatorname{ker}\left(\mathcal{O}_{n}(q)\right) \backslash\{0\}$, we have $y\left(r, x_{0}, u\right)=y(r, 0, u)$ for any $u$ when $r \equiv q$. Assume now that $q$ and $q^{\prime}$ are neither controlled-discernible nor jointly observable. Then $\Gamma_{N}(q)-\Gamma_{N}\left(q^{\prime}\right)=$ 0 for all $N$ and, letting $r \equiv q$ and $r^{\prime} \equiv q^{\prime}$, we get

$$
y\left(r, x_{0}, u\right)-y\left(r^{\prime}, x_{0}^{\prime}, u\right)=y\left(r, x_{0}, 0\right)-y\left(r^{\prime}, x_{0}^{\prime}, 0\right),
$$

and so, by definition of joint observability, there exist two initial states $x_{0}$ and $x_{0}^{\prime}$ such that $y\left(r, x_{0}, 0\right) \neq y\left(r^{\prime}, x_{0}^{\prime}, 0\right)$, and by (60), such that $y\left(r, x_{0}, u\right) \neq y\left(r^{\prime}, x_{0}^{\prime}, u\right)$ for all controls $u$.

$3 \Rightarrow 2$ : We need to show that the set of controls

$$
\mathcal{U} \triangleq\left\{u \in \mathcal{A} \mid \forall r, \forall r^{\prime}, \forall x_{0}, \forall x_{0}^{\prime}, x_{0}^{\prime} \neq x_{0} \Rightarrow y\left(r, x_{0}, u\right) \neq y\left(r^{\prime}, x_{0}^{\prime}, u\right)\right\}
$$

is generic if condition 3. holds. Let us show that it in fact contains

$\mathcal{U}_{4 n} \triangleq\left\{u \in \mathcal{A} \mid u_{0}^{[4 n]} \in \mathbb{R}^{4 m n} \backslash \bigcup_{\left(q, q^{\prime}\right) \in S} \operatorname{ker}\left(\left(I-P_{4 n}\left(q, q^{\prime}\right)\right)\left(\Gamma_{4 n}(q)-\Gamma_{4 n}\left(q^{\prime}\right)\right)\right)\right\}$,

where $S$ is the set of controlled-discernible pairs of modes. By proposition 3 . $\mathcal{U}_{4 n}$ is of course generic. Now, take $x_{0} \neq x_{0}^{\prime}, r, r^{\prime}$ and $T>0$ such that $r_{[1, T]} \equiv q$ and $r^{\prime}[0, T] \equiv q^{\prime}$. If $q=q^{\prime}$, then of course $y\left(r, x_{0}, u\right) \neq y\left(r^{\prime}, x_{0}^{\prime}, u\right)$ for all $u \in \mathcal{A}$ since $q$ is observable. If $q \neq q^{\prime}$ and they are controlled-discernible, then by Lemma 2 and Proposition [3. $y_{[0, T]}\left(r, x_{0}, u\right) \neq y_{[0, T]}\left(r^{\prime}, x_{0}^{\prime}, u\right)$ if $u \in$ $\mathcal{U}_{4 n}$. If they are not controlled-discernible, then they are jointly observable and so $y_{[0, T]}\left(r, x_{0}, u\right) \neq y_{[0, T]}\left(r^{\prime}, x_{0}^{\prime}, u\right)$ for all $u \in \mathcal{A}$ since $y_{[0, T]}\left(r, x_{0}, u\right)-$ $y_{[0, T]}\left(r^{\prime}, x_{0}^{\prime}, u\right)=y_{[0, T]}\left(r, x_{0}, 0\right)-y_{[0, T]}\left(r^{\prime}, x_{0}^{\prime}, 0\right)$.

Remark 3. What we have just shown is that for switched linear systems with arbitrary and unknown mode signals, single-experiment observability and generic single-experiment observability, as defined in [17, are equivalent, whether one wishes to observe the initial mode or the initial state.

Remark 4. In [7, recall that a necessary and sufficient condition for $\left(r_{0}, x_{0} / y, u^{*}\right)$-observability was established as the combination of controlleddiscernibility of each pair of modes and observability of each mode. While 
controlled-discernibility of every pair of modes is indeed necessary and sufficient for $\left(r_{0} / y, u^{*}\right)$-observability, we have established that it is not necessary for $\left(x_{0} / y, u^{*}\right)$-observability. Indeed, as noted in Remark 1, the system given in Example 1 is $\left(x_{0} / y, u^{*}\right)$-observable even though $\Gamma_{N}(q)=0$ for any mode, making controlled-discernibility an impossibility. Informally, it shows that it is not necessary to recover the initial mode in order to figure out the initial state.

\section{Conclusion}

We have characterized several observability notions for continuous-time switched linear systems. The analysis is of course still incomplete, and several problems still need to be solved. For instance, mode and state observability properties under fully or partially unknown inputs still have not been investigated in the switched setting. Furthermore, we will be investigating the existential counterparts of our current results, i.e. conditions for existence of mode signals allowing the initial or current mode or state to be inferred. It turns out that, in contrast with the universal problems that reduce to instantaneous inversions, such problems will involve observing the outputs over a period of time, and will involve the design of switching signals (as pointed out, e.g., in [22], and as is the case in the known modes case [19]). In future work, we will furthermore explore the connection between observability and bisimulation theory for discrete event and hybrid systems [16, 20].

\section{Acknowledgements}

The authors wish to thank the anonymous reviewers for their careful reviews and insightful comments, as well as Hakan Yazarel for valuable discussions.

\section{References}

1. M. Babaali and M. Egerstedt, "Observability of switched linear systems." in Hybrid Systems: Computation and Control (R. Alur and G. Pappas, eds.). Springer, 2004, pp. $48-63$.

2. A. Balluchi, L. Benvenuti, M. D. Di Benedetto, and A. L. Sangiovanni-Vincentelli, "Observability for hybrid systems," in Proceedings of the 42nd IEEE Conference on Decision and Control, Maui, HW, December 2003.

3. A. Bemporad, G. Ferrari-Trecate, and M. Morari, "Observability and controllability of piecewise affine and hybrid systems," IEEE Transactions on Automatic Control, vol. 45, no. 10, pp. 1864-1876, October 2000.

4. S. L. Campbell and C. D. J. Meyer, Generalized Inverses of Linear Transformations. New York, NY: Dover, 1991.

5. P. Collins and J. H. van Schuppen, "Observability of piecewise-affine hybrid systems," ser. Hybrid Systems: Computation and Control. Springer-Verlag, 2004.

6. E. F. Costa and J. B. R. do Val, "On the detectability and observability of discretetime Markov jump linear systems," in Proceedings of the 39th IEEE Conference on Decision and Control, Sydney, Australia, December 2000, pp. 2355-2360. 
7. E. De Santis, M. D. Di Benedetto, and G. Pola, "On observability and detectability of continuous-time linear switching systems," in Proceedings of the 42nd IEEE Conference on Decision and Control, Maui, HW, December 2003.

8. J. Ezzine and A. H. Haddad, "Controllability and observability of hybrid systems," International Journal of Control, vol. 49, no. 6, pp. 2045-2055, 1989.

9. G. Ferrari-Trecate and M. Gati, "Computation of observability regions for discretetime hybrid systems," in Proceedings of the 42nd IEEE Conference on Decision and Control, Maui, HW, December 2003.

10. L. Gurvits, "Stabilities and controllabilities of switched systems (with applications to the quantum systems)," in Proceedings of the Fifteenth International Symposium on Mathematical Theory of Networks and Systems, Univ. Notre Dame, August 2002.

11. I. Hwang, H. Balakrishnan, and C. Tomlin, "Observability criteria and estimator design for stochastic linear hybrid systems," in Proceedings of the IEE European Control Conference, Cambridge, UK, September 2003.

12. Y. Ji and H. Chizeck, "Controllability, observability and discrete-time jump linear quadratic control," International Journal of Control, vol. 48, no. 2, pp. 481-498, 1988.

13. A. Juloski, M. Heemels, and S. Weiland, "Observer design for a class of piecewise affine systems," in Proceedings of the 41st IEEE Conference on Decision and Control, Las Vegas, NV, December 2002.

14. T. Kailath, Linear Systems. Englewood Cliffs, NJ: Prentice Hall, 1980.

15. M. Mariton, "Stochastic observability of linear systems with markovian jumps," in Proceedings of the 25th IEEE Conference on Decision and Control, Athens, Greece, December 1986, pp. 2208-2209.

16. G. J. Pappas, "Bisimilar linear systems," Automatica, vol. 39, no. 12, pp. 20352047, December 2003.

17. E. D. Sontag, "On the observability of polynomial systems, I: Finite-time problems," SIAM Journal on Control and Optimization, vol. 17, no. 1, pp. 139-151, 1979.

18. — - "Nonlinear regulation: The piecewise linear approach," IEEE Transactions on Automatic Control, vol. 26, no. 2, pp. 346-358, April 1981.

19. Z. Sun, S. S. Ge, and T. H. Lee, "Controllability and reachability criteria for switched linear systems," Automatica, May 2002.

20. P. Tabuada and G. J. Pappas, "Bisimilar control affine systems," Systems \& Control Letters, vol. 52, no. 1, pp. 49-58, May 2004.

21. R. Vidal, A. Chiuso, and S. Soatto, "Observability and identifiability of jump linear systems," in Proceedings of the 41st IEEE Conference on Decision and Control, Las Vegas, NV, December 2002, pp. 3614-3619.

22. R. Vidal, A. Chiuso, S. Soatto, and S. Sastry, "Observability of linear hybrid systems," ser. Hybrid Systems: Computation and Control. Springer-Verlag, 2003.

23. P. D. West and A. H. Haddad, "On the observability of linear stochastic switching systems," in Proceedings of the 1994 American Control Conference, Baltimore, MD, June 1994, pp. 1846-1847. 